\title{
Mammary analogue secretory carcinoma of a salivary gland of the hard palate with contralateral cervical lymph node metastases: A case report
}

\author{
ANTON STRAUB ${ }^{1}$, CHRISTIAN LINZ $^{1}$, SABRINA STROBEL ${ }^{2}$, STEFAN HARTMANN ${ }^{1}$, JULIAN HOHM ${ }^{1}$, \\ ANDREAS FUCHS $^{1}$, URS MÜLLER-RICHTER ${ }^{1}$, ALEXANDER KÜBLER ${ }^{1}$ and ROMAN BRANDS ${ }^{1}$ \\ ${ }^{1}$ Department of Maxillofacial and Plastic Surgery of The University of Wuerzburg, D-97070 Wuerzburg; \\ ${ }^{2}$ Institute of Pathology of The University of Wuerzburg, D-97080 Wuerzburg, Germany
}

Received February 5, 2021; Accepted June 1, 2021

DOI: $10.3892 / \mathrm{mco} .2021 .2389$

\begin{abstract}
Mammary analogue secretory carcinoma (MASC) is a rare malignant tumour of the salivary glands, with only few cases reported in the literature to date. Initial preoperative staging is crucial for all patients with an oral malignancy to visualize the tumour, detect lymph node or distant metastases and plan therapeutic interventions. In the case presented herein, radiological imaging revealed a tumour of the right hard palate with suspected positive contralateral lymph nodes. Therefore, local tumour resection comprising hemimaxillectomy and bilateral neck dissection was performed. The diagnosis of MASC was finally based on characteristic histopathological and immunohistochemical findings, such as S100 protein and mammaglobin positivity. The diagnosis of MASC may be challenging, as such findings lack specificity. To confirm the diagnosis, molecular genetic examinations may be performed to detect a highly specific ETV6-NTRK3 fusion gene. Depending on the results of these examinations, surgery, alone or combined with adjuvant radiation or chemoradiation, is the recommended approach. In summary, MASC should be treated similarly to other low-grade salivary gland tumours, such as acinic cell carcinoma, as they exhibit biological and histopathological similarities.
\end{abstract}

Correspondence to: Dr Anton Straub, Department of Maxillofacial and Plastic Surgery of The University of Wuerzburg, Pleicherwall 2, D-97070 Wuerzburg, Germany

E-mail: straub_a@ukw.de

Abbreviations: AC, adenocarcinoma; AciCC, acinic cell carcinoma; CAC, cystadenocarcinoma; FDG, $\left[{ }^{18} \mathrm{~F}\right]$-fluorodeoxyglucose; FISH, fluorescence in situ hybridization; MASC, mammary analogue secretory carcinoma; MuC, mucoepidermoid carcinoma; PET, positron emission tomography; TRKI, tyrosine kinase inhibitor; US, ultrasound

Key words: mammary analogue secretory carcinoma, salivary gland cancer, neck dissection, oral cancer, oral cavity, lymph node metastases, head and neck, cancer

\section{Introduction}

Mammary analogue secretory carcinoma (MASC) is a rare tumour of the head and neck region first described in 2010 by Skálová et al (1). As of 2017, MASC has been listed in the Classification of Head and Neck Tumours by the World Health Organization (2). MASC is a malignancy of the salivary glands, predominantly observed in the parotid gland, with an incidence rate of $4-4.5 \%$ among all malignant salivary gland tumours (3-5). However, when considering only tumours of the small salivary glands, the incidence of MASC is markedly lower (4). MASC usually occurs at a mean age of 45 years and is slightly more common among men (6).

To diagnose MASC, microscopic examination is necessary. On histopathological examination, the tumour typically displays cystic, tubular and solid areas with infiltration. Most MASCs consist of relatively monomorphic cells with a moderate amount of vacuolated eosinophilic cytoplasm. On immunohistochemical examination, the tumour shows immunoreactivity for S-100, mammaglobin, MUC4 and CK7 (3). Mammaglobin and S100 protein show high sensitivity (95\%), but are not specific for MASC (6-8). By contrast, an ETV6-neurotrophic receptor tyrosine kinase (NTRK)3 fusion gene is highly specific for MASC, at least considering salivary gland malignancies. This translocation can be detected by fluorescence in situ hybridization (FISH) or PCR analysis and confirms the diagnosis of MASC (1,3,9-11). In addition to NTRK1 and NTRK2, NTRK3 is a membrane-bound receptor and part of the NTRK neurotrophin receptor family, which is involved in neuronal cell differentiation and proliferation. Mutations play an important role in carcinogenesis, such as that in secretory breast carcinomas, medulloblastomas and MASCs (11). Approximately 300 cases of MASC have been reported in the literature to date. Due to this limited number of cases, no consistent data concerning treatment procedures and outcomes have been published. The majority of MASCs are considered as low-grade malignancies, they are treated by radical resection and have a good prognosis (10). According to the previously reported cases, patients with MASC have a 5-year overall survival rate of $95 \%$ and a 5 -year disease-free survival rate of $89 \%$, with a 
low incidence of nodal metastases (12). However, cases with high-grade transformation, lymph node and distant metastases have rarely been described (13). The case of a patient with MASC of the hard palate with bony infiltration and contralateral cervical lymph node metastases is described in the present study. This combination is extraordinarily rare and, to the best of our knowledge, has not been described in the literature to date (5). The aim of the present study was to focus on treatment procedures recommended for high-risk patients, discuss the benefits of selective neck dissection and adjuvant chemoradiation, and discuss novel treatment options with entrectinib, larotrectinib and other tyrosine kinase inhibitors (TRKIs) for patients with inoperable tumours or under palliative care.

\section{Case report}

A 44-year-old Caucasian woman initially presented at the Department of Oral and Maxillofacial Plastic Surgery of the University Hospital of Wuerzburg (Wuerzburg, Germany) in June 2018. The patient reported painless swelling in the area of the right hard palate (Fig. 1). A biopsy had already been performed at a private practice office, and histopathological examination revealed a carcinoma with immunoreactivity for CK7, S100 and mammaglobin. No irregularities and no radiotherapy of the head and neck region were noted in the patient's history.

The staging procedures included clinical examination, ultrasound (US) of the neck, MRI, cone beam CT and $\left[{ }^{18} \mathrm{~F}\right]$-fluorodeoxyglucose-positron emission tomography/CT (FDG-PET/CT).

The MRI examination revealed a tumour measuring 18x16x12 mm, with invasion of the right maxillary sinus and suspected retropharyngeal and cervical lymph nodes on the left side on FDG-PET/CT. By contrast, CT and MRI showed bilateral suspected lymph nodes (Figs. 2-4). A secondary suspected pharyngeal lesion at the tongue base was also identified.

Based on the aforementioned findings, the patient underwent local resection of the hard palate tumour, with a clinical safety distance of $10 \mathrm{~mm}$, along with bilateral radical neck dissection (levels I-V) with temporary tracheotomy. Plastic reconstruction of the hard palate was performed during the same operation using a radial forearm flap. Biopsies of the suspected lesion in the pharynx showed no signs of malignancy. Histopathological examination of the specimen revealed a tumour with a diameter of $13 \mathrm{~mm}$, with the closest margins at $3 \mathrm{~mm}$, medullary bone invasion and two (2/37) lymph node metastases on the contralateral side of the neck at level Va. No metastases were detected on the ipsilateral side of the neck. Histopathological examination of the tumour revealed microcystic, tubular and solid areas with infiltration. The tumour cells were monomorphic and round-oval, with a moderate amount of vacuolated eosinophilic cytoplasm (Figs. 5 and 6). On immunohistochemical examination, the tumour was positive for CK7, S100, mammaglobin, GATA3, GCDF-P15 and pan-cytokeratin (AE1/3). P16 expression in tumour cells, partial reactivity of SOX10 and specific reactivity for CD117 were also detected. The Ki-67 index was low $(<5 \%)$. FISH analysis performed using an ETV6 break-apart

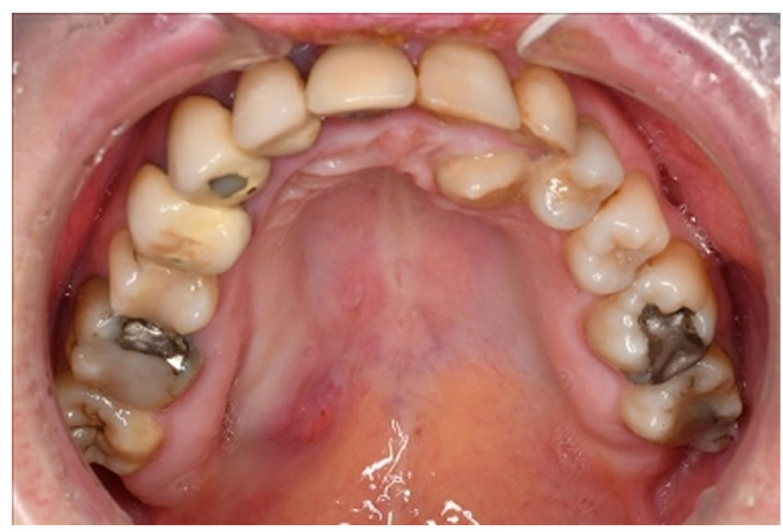

Figure 1. Painless swelling $(\sim 1 \mathrm{x} 1 \mathrm{~cm})$ in the right hard palate with ulceration due to the biopsy performed in the private surgery practice. Apart from the lesion, the oral mucosa appeared normal. Dental status: Tooth 23 was retained. Apart from that finding, the dental chart was inconspicuous, without any signs of loosening teeth.

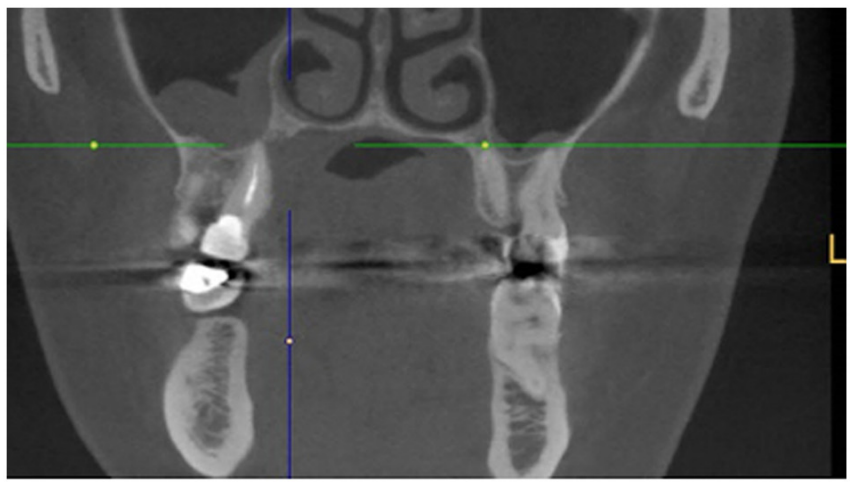

Figure 2. Cone beam CT revealed bone invasion in the right hard palate. The mucosa of the right maxillary sinus exhibited reactive changes.

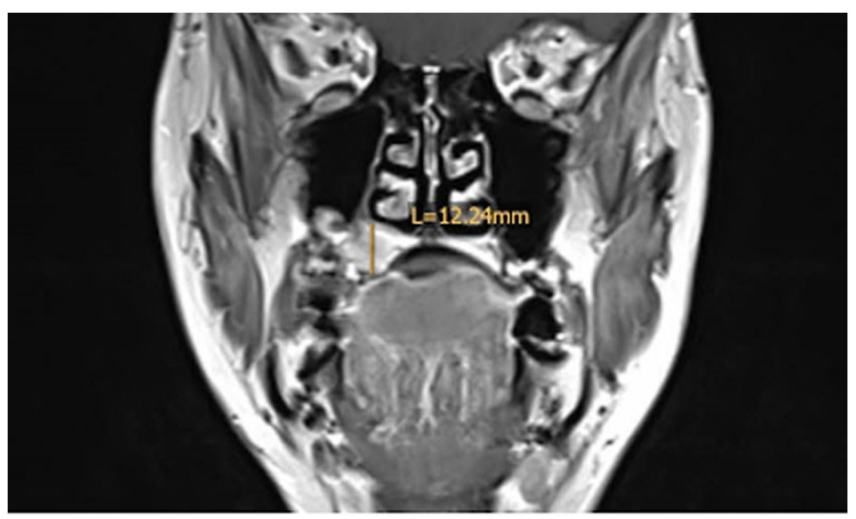

Figure 3. MRI examination revealed a tumour measuring $18 \times 16 \times 12 \mathrm{~mm}$ in the right hard palate. Suspected lymph nodes were observed on both sides of the neck.

probe was positive. Considering the findings of histopathological and immunohistochemical examinations, and the FISH results with proof of a break in the ETV6-gene (12q13), the diagnosis of MASC was confirmed (Figs. 5-8). Based on these findings, the tumour stage was defined as follows: pT4a, pN2c (2/37), pL0, pV0 and pPn0 (8th Edition of the 


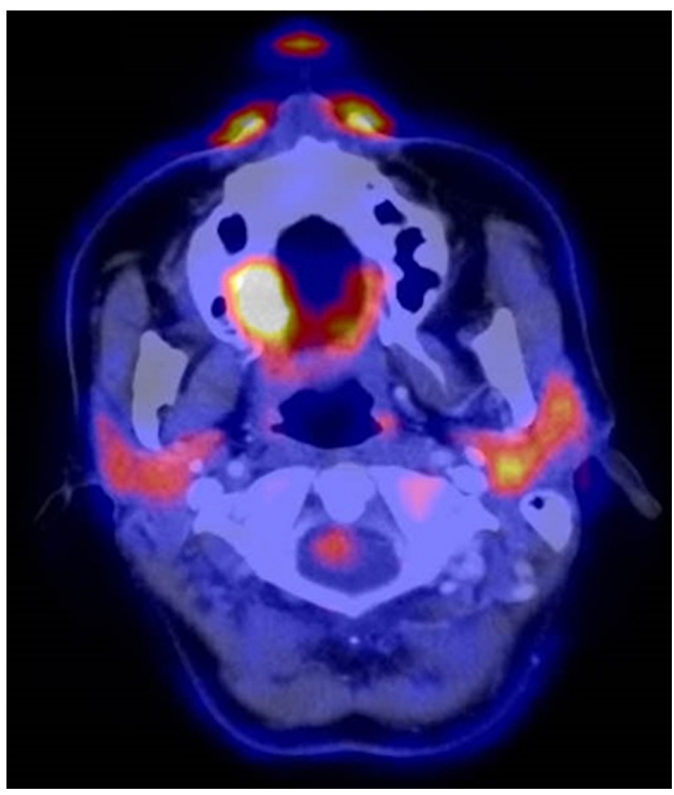

Figure 4. Positron emission tomography-CT revealed an FDG-avid tumour mass in the right hard palate and contralateral cervical lymph node metastases. A second FDG-avid lesion in the pharynx exhibited no sign of malignancy on biopsy. FDG, $\left[{ }^{18} \mathrm{~F}\right]$-fluorodeoxyglucose.

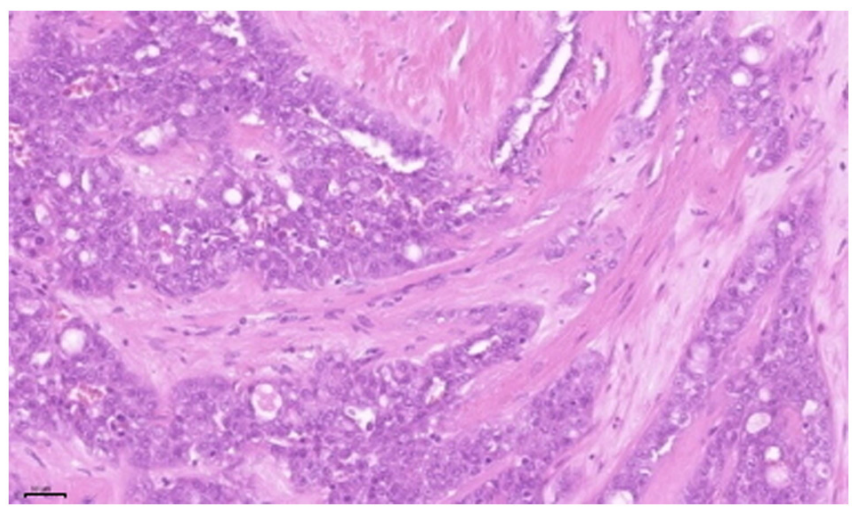

Figure 5. HE staining of the resectate (magnification, $\mathrm{x} 20$; scale bar, $50 \mu \mathrm{m}$ ). On microscopic examination, the tumour was solid, microcystic and tubular, with fibrous septa. The tumour cells appeared monomorphic and round-oval, with small nucleoli and fine granular chromatin. The cytoplasm was weakly eosinophilic and partly vacuolated.

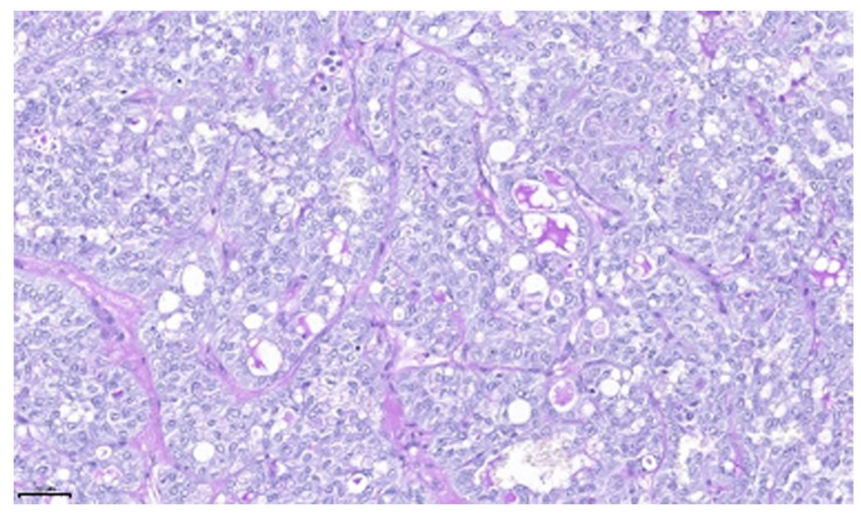

Figure 6. PAS staining of the resectate (magnification, $\mathrm{x} 20$; scale bar, $50 \mu \mathrm{m}$ ). Eosinophilic and PAS-positive secretions are evident in the microcystic areas. PAS, Periodic acid-Schiff.
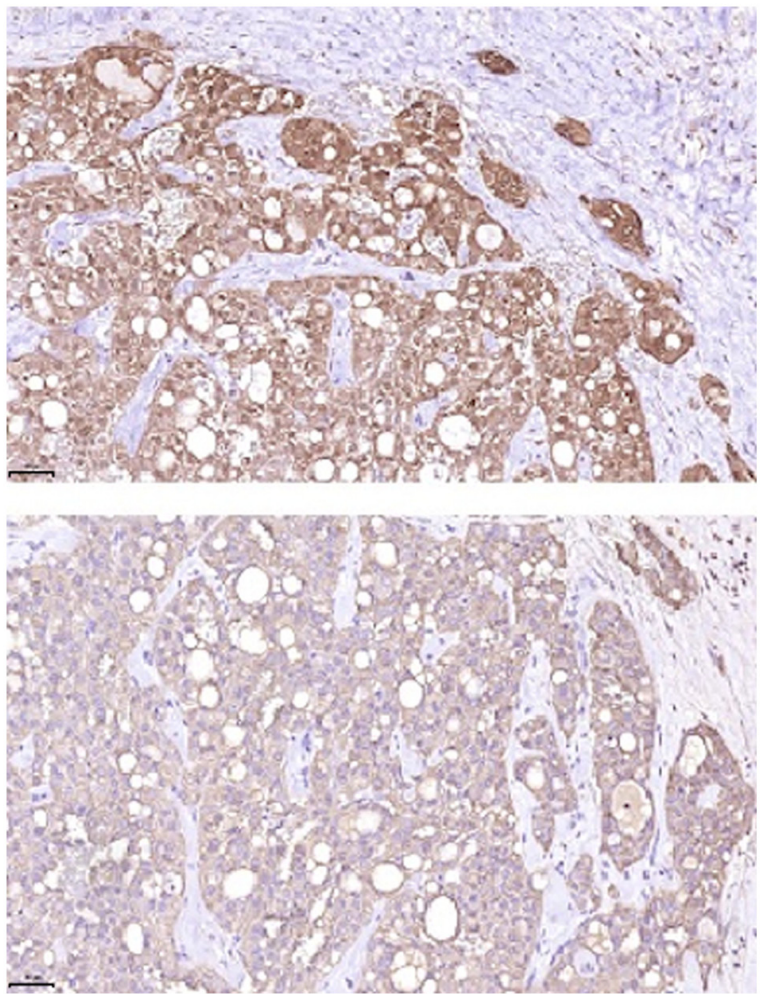

Figure 7. Immunohistochemical examination of the resectate (magnification, $\mathrm{x} 50$; scale bar, $50 \mu \mathrm{m}$ ) revealed specific positivity for $\mathrm{S} 100$ (top panel) and specific cytoplasmic positivity for mammaglobin (bottom panel).

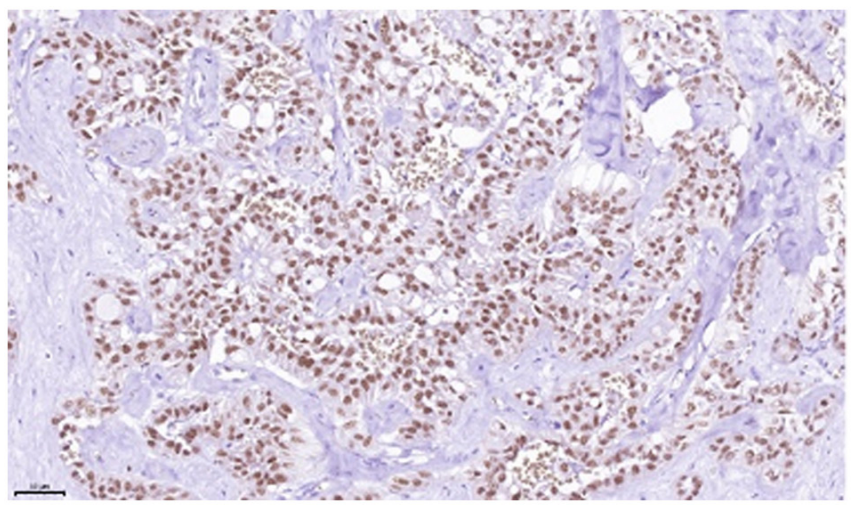

Figure 8. Immunohistochemical examination of the resectate (magnification, $\mathrm{x} 50$; scale bar, $50 \mu \mathrm{m}$ ) revealed specific nuclear positivity for GATA3.

TNM Classification, 2017) (14). Due to the several high-risk characteristics of this tumour, including bone invasion and contralateral nodal metastases, adjuvant radiochemotherapy with 30 rounds of radiation (dynamic volumetric modulated arc therapy; 54.00/66.00 Gy) and cisplatin (a cumulative dose of $200 \mathrm{mg} / \mathrm{m}^{2}$ body surface area) was performed. A radiation dose of 54.00 Gy was delivered to the area of lymphatic cervical drainage, and 66 Gy was delivered to the area of the tumour in the hard palate.

The postoperative course was uneventful, and the patient has visited our outpatient centre regularly to date (last follow-up visit, March 2021), without signs of recurrence. The postoperative clinical examination revealed limited abduction 
of the shoulder and limited opening of the mouth, for which physiotherapy and exercises were performed.

\section{Discussion}

MASC is a rare type of head and neck cancer that mostly affects the major salivary glands. As a malignant tumour, preoperative staging examinations are necessary, including clinical examination, radiological imaging, and histological, immunohistochemical and molecular genetic examinations.

In the case presented herein, preoperative imaging revealed a tumour measuring 18x16x12 mm, with bony infiltration of the right hard palate and cervical lymph node metastases. Suspected lymph node metastases were shown on both sides of the neck on MRI and CT scans. By contrast, FDG-PET/CT scan only showed suspected lymph nodes on the contralateral side of the neck. There were no signs of distant metastases.

Histological examination revealed a malignant tumour of a minor salivary gland, and immunohistochemical examinations confirmed the diagnosis of MASC by showing the typical characteristics of this entity. To verify the diagnosis of MASC, several differential diagnoses had to be excluded. In a retrospective review, Chiosea et al (15) reported that 11 of $89(12.4 \%)$ patients with MASC were incorrectly diagnosed with acinic cell carcinoma (AciCC), and 14 of $37(37.8 \%)$ patients were incorrectly diagnosed with adenocarcinoma (AC). In particular, MASC and AciCC share histological similarities. Although AciCC is characterized by basophilic cytoplasm and zymogen granules, MASC cells typically have eosinophilic cytoplasm and no zymogen granules, which was consistent with the histopathological findings of the present case. However, zymogen-poor AciCCs are very difficult to differentiate from MASCs $(1,6,16)$. Strong S100 protein and mammaglobin expression levels may help differentiate between the two entities, as these findings exclude AciCC $(6,10,17)$. Another important differential diagnosis of MASC is AC, which can express S100 and mammaglobin and is typically observed in the minor glands (6). Other differential diagnoses, such as cystadenocarcinoma (CAC) and mucoepidermoid carcinoma (MuC), must also be considered (6). Due to these findings in some cases, histopathological and immunohistochemical examinations do not ensure the accurate diagnosis of MASC. In these cases, detection of the $\mathrm{t}(12 ; 15)(\mathrm{p} 13 ; \mathrm{q} 25)$ translocation (ETV6-NTRK3 fusion gene) may help differentiate between MASC and other similar tumours $(3,6)$. The ETV- 6 gene encodes a tyrosine kinase that regulates cell proliferation and differentiation. Considering only salivary gland neoplasms, this translocation is highly specific for MASC $(3,7)$. In the case presented herein, immunohistochemical and molecular genetic examinations confirmed the diagnosis of MASC.

As the existing literature is limited, no consistent therapy guidelines are currently available for this tumour entity. In the literature, the majority of the patients received treatment based on histochemical similarities and similar growth patterns analogous to other low-grade malignancies or AciCC, and underwent surgery with or without adjuvant radiation or chemoradiation $(6,10,13)$. In a review of the literature, primary radiotherapy or chemoradiation were not considered as common therapeutic options (17). Therefore, only one case report was found concerning a patient with MASC who was treated with primary radiotherapy. That patient had a locally advanced MASC of the parotid gland, without nodal or distant metastases. The patient received radiation (66 Gy) with cetuximab and achieved stable disease. Cetuximab is an EGFR-TRKI which, along with other TRKIs, may be useful for treating MASC (18).

For sufficient tumour resection, a clinical safety margin of $10 \mathrm{~mm}$ is considered to be appropriate, and it is obligatory in head and neck squamous cell carcinoma according to German guidelines (19). Currently, no valid data are available on whether selective neck dissection in patients with a negative nodal status improves patient outcomes and overall survival. In the present case, two nodal metastases were identified at level $\mathrm{Va}$ on the contralateral side of the neck, although MASC is normally a low-grade malignancy and nodal metastases are rare. Chiosea et al (15) reported cervical nodal metastases in $6 / 34$ patients $(17.6 \%)$. In another study, 4/18 (22\%) patients had nodal metastases (6), which were mostly observed in patients with a higher $\mathrm{T}$ stage (T3 or T4) and submandibular gland tumours (17). Owing to the small number of cases in those studies, a distinct recommendation for neck dissection cannot be given. However, the data indicate that patients may benefit from selective neck dissection, even when imaging examinations are negative for nodal metastases. Levels I, II, III and Va are more likely to be affected by nodal metastases from MASC, similar to other oral malignancies $(20,21)$. For cases with a positive nodal status, uni- or bilateral neck dissection of levels I-V should be performed, and adjuvant radiation or chemoradiation is necessary due to the more aggressive tumour behaviour (22). In cases of a low-grade malignancy without nodal metastases, surgery alone may be sufficient (6). Based on the MRI and $\mathrm{CT}$ findings in the present case, bilateral radical neck dissection was performed. Histopathological examination of the resectate detected two (2/37) lymph node metastases corresponding to the lesions on FDG-PET/CT on the contralateral, but not the ipsilateral, side of the neck. Lymph nodes on the ipsilateral side may have appeared enlarged and suspicious on MRI and CT due to the preoperative biopsy performed in a private practice. Of note, a biopsy should be preferably performed after imaging examinations to prevent the detection of false-positive lymph nodes and overtreatment (23). In the present case, nodal dissection of the lower ipsilateral levels may have been prevented by this approach, further demonstrating the higher specificity of FDG-PET/CT for detecting cervical nodal metastases compared with CT and MRI (24,25). Currently, FDG-PET/CT is not routinely performed in patients with head and neck cancer due to the lack of clear recommendations, limited availability and high cost.

Due to the positive nodal status, the patient received adjuvant chemoradiation with cisplatin and $66 \mathrm{~Gy}$. It is unclear whether chemoradiation is superior to radiation. In a study by Sethi et al (17), only 2/86 (2.3\%) of patients with MASC received adjuvant chemotherapy, in contrast to $21 / 86(24.4 \%)$ patients who received radiation without chemotherapy. This approach is supported by a review in 2019 comparing the treatments received by 7,342 patients with salivary gland cancer. The patients were separated into different subgroups 
by histological subtype. The patients had AciCC (20.6\%) and mucoepidermoid carcinoma (36.4\%), but not MASC. The treatment differed significantly across the subgroups. Overall, $42 \%$ of the patients with AciCC received surgery and adjuvant radiation, and only $4 \%$ received surgery and adjuvant chemoradiation. In this subgroup, the 2- and 5-year overall survival rates for surgery plus radiotherapy vs. surgery plus chemoradiation were 95 and $84 \%$ vs. 81 and 63\%, respectively (26). If these data are extrapolated to MASC, the treatment for which is similar to that for AciCC, there is no scientific evidence supporting the benefit of adjuvant chemotherapy. Thus, adjuvant radiation appears to be appropriate for high-risk patients with nodal metastases.

Selective TRKI treatment may also be considered for therapy in patients with locally advanced, metastatic or inoperable tumours (27). For example, TRKIs as a treatment option was reported in a clinical study of 55 patients with TRK fusion-positive cancers. MASC was identified in 12 of these patients $(21.81 \%)$ and was the most frequent entity. All the patients had advanced local tumour or metastases, or had already received standard therapy; therefore, they were treated with the TRKI larotrectinib. Larotrectinib at $100 \mathrm{mg}$ was administered twice per day until tumour progression, the occurrence of adverse events, or withdrawal from the study. Of the 55 patients, $7(13 \%)$ had a complete response, $34(62 \%)$ had a partial response, $7(13 \%)$ had stable disease, 5 (9\%) had progressive disease, and 2 (4\%) could not be evaluated. The overall response rate was $80 \%$. No difference in therapy efficiency was observed across the subgroups. Of note, of the 12 patients with MASC, only 1 had progressive disease, while the remaining 11 patients exhibited a partial or complete response to larotrectinib therapy. Consistently, the European Medicines Agency approved larotrectinib for the treatment of solid tumours with NTRK gene fusion, including MASC. Another potential treatment option is entrectinib, which has also been approved for the treatment of TRK-expressing tumours. Several phase 1 and 2 studies, such as ALKA, STARTRK-1 and STARTRK-2, are investigating the potential benefits of entrectinib for the treatment of cancers with detected molecular alterations in the TRK1, TRK2 and TRK3 genes (28). STARTRK-2, in particular, includes patients with salivary gland tumours harbouring a NTRK3 gene fusion, similar to that in MASC. In addition to entrectinib and larotrectinib, which are two approved therapy options, other TRKIs, such as sitravatinib, cabozantinib, belizatinib, and several more, have been evaluated in clinical trials (29).

These data indicate a potential therapy option for patients with relapse after standard therapy, patients with inoperable tumours (locally advanced and/or metastatic), or those under palliative care (30), and underscore the significance of molecular diagnostic methods to detect the ETV6-NTRK3 fusion gene. It may be of value to re-evaluate whether this fusion gene is present in patients with inoperable salivary gland tumours and those under palliative care. This approach appears to be appropriate, as MASC is often initially misdiagnosed as AciCC, AC, CAC or MuC; thus, molecular diagnosis may uncover new therapy options.

Adequate follow-up must be applied postoperatively. A mean follow-up period of 5 years with imaging examinations at specific intervals appears to be appropriate. We suggest that the first imaging examination be performed 6 months after surgery, followed by imaging once per year until follow-up is completed. In the first 2 years and in high-risk patients the intervals between assessments should be shorter (every 3-4 months).

This case underscores the need for sufficient staging examinations and accurate diagnosis, which are crucial for selecting the optimal therapy procedures and may uncover different (molecular) treatment options for patients receiving palliative care. If a local tumour without nodal or distant metastases is detected, surgery alone should be performed. However, when aggressive tumour behaviour and nodal metastases are observed, selective neck dissection should be considered, even when the nodal status is negative. If the nodal status is positive, ipsi- or bilateral neck dissection of levels I-V and adjuvant radiation or chemoradiation are necessary. The benefits of chemotherapy remain unclear. New molecular targeted therapies with TRKIs, such as larotrectinib and entrectinib, may be promising treatment options for patients with advanced inoperable disease and underscore the need for molecular diagnosis in selected cases.

\section{Acknowledgements}

Not applicable.

\section{Funding}

No funding was received.

\section{Availability of data and materials}

Clinical data and complementary examinations are available from the corresponding author on reasonable request.

\section{Authors' contributions}

AS, RB and SH accrued all data, obtained the patient's informed consent and drafted the manuscript. AS, RB and AK have seen and can confirm the authenticity of the raw data. SS provided the histopathological information and images of the obtained resectate. All the authors have contributed to writing the manuscript. AS, RB, JH, CL, AF and UMR prepared the final version of the manuscript. All the authors have read and approved the final manuscript.

\section{Ethics approval and consent to participate}

Not applicable.

\section{Patent consent for publication}

Written informed consent was obtained from the patient regarding the publication of the case details and any accompanying images.

\section{Competing interests}

The authors declare that they have no competing interests. 


\section{References}

1. Skálová A, Vanecek T, Sima R, Laco J, Weinreb I, Perez-Ordonez B, Starek I, Geierova M, Simpson RH, Passador-Santos F, et al: Mammary analogue secretory carcinoma of salivary glands, containing the ETV6-NTRK3 fusion gene: A hitherto undescribed salivary gland tumor entity. Am J Surg Pathol 34: 599-608, 2010.

2. Seethala RR and Stenman G: Update from the 4th Edition of the World Health Organization classification of head and neck tumours: Tumors of the salivary gland. Head Neck Pathol 11: 55-67, 2017.

3. Luk PP, Selinger CI, Eviston TJ, Lum T, Yu B, O'Toole SA, Clark JR and Gupta R: Mammary analogue secretory carcinoma: An evaluation of its clinicopathological and genetic characteristics. Pathology 47: 659-666, 2015.

4. Majewska H, Skalova A, Stodulski D, Klimková A, Steiner P, Stankiewicz C and Biernat W: Mammary analogue secretory carcinoma of salivary glands: A new entity associated with ETV6 gene rearrangement. Virchows Arch 466: 245-254, 2015.

5. Boliere C, Murphy J, Qaisi M, Manosca F and Fung H: Mammary analogue secretory carcinoma of the palate: Case report and review of the literature. Case Rep Dent 2019: 7416302, 2019.

6. Stevens TM and Parekh V: Mammary analogue secretory carcinoma. Arch Pathol Lab Med 140: 997-1001, 2016.

7. Shah AA, Wenig BM, LeGallo RD, Mills SE and Stelow EB Morphology in conjunction with immunohistochemistry is sufficient for the diagnosis of mammary analogue secretory carcinoma. Head Neck Pathol 9: 85-95, 2015.

8. Patel KR, Solomon IH, El-Mofty SK, Lewis JS Jr and Chernock RD: Mammaglobin and S-100 immunoreactivity in salivary gland carcinomas other than mammary analogue secretory carcinoma. Hum Pathol 44: 2501-2508, 2013.

9. Khalele BA: Systematic review of mammary analog secretory carcinoma of salivary glands at 7 years after description. Head Neck 39: 1243-1248, 2017.

10. Skalova A: Mammary analogue secretory carcinoma of salivary gland origin: An update and expanded morphologic and immunohistochemical spectrum of recently described entity. Head Neck Pathol 71 (Suppl 1): S30-S36, 2013.

11. Luo Y, Kaz AM, Kanngurn S, Welsch P, Morris SM, Wang J, Lutterbaugh JD, Markowitz SD and Grady WM: NTRK3 is a potential tumor suppressor gene commonly inactivated by epigenetic mechanisms in colorectal cancer. PLoS Genet 9: e1003552, 2013

12. Boon E, Valstar MH, van der Graaf WTA, Bloemena E, Willems SM, Meeuwis CA, Slootweg PJ, Smit LA, Merkx MAW, Takes RP, et al: Clinicopathological characteristics and outcome of 31 patients with ETV6-NTRK3 fusion gene confirmed (mammary analogue) secretory carcinoma of salivary glands. Oral Oncol 82: 29-33, 2018.

13. Skálová A, Vanecek T, Majewska H, Laco J, Grossmann P, Simpson RH, Hauer L, Andrle P, Hosticka L, Branžovský J and Michal M: Mammary analogue secretory carcinoma of salivary glands with high-grade transformation: Report of 3 cases with the ETV6-NTRK3 gene fusion and analysis of TP53, $\beta$-catenin, EGFR, and CCND1 genes. Am J Surg Pathol 38: 23-33, 2014.

14. Brierley J, Gospodarowicz $M$ and Wittekind C: Head and Neck Tumours, Lip and Oral Cavity. In: TNM Classification of Malignant Tumours. 8th edition. Wiley-Blackwell, Toronto, pp17-54, 2017.

15. Chiosea SI, Griffith C, Assaad A and Seethala RR Clinicopathological characterization of mammary analogue secretory carcinoma of salivary glands. Histopathology 61 : 387-394, 2012

16. Connor A, Perez-Ordonez B, Shago M, Skálová A and Weinreb I: Mammary analog secretory carcinoma of salivary gland origin with the ETV6 gene rearrangement by FISH: Expanded morphologic and immunohistochemical spectrum of a recently described entity. Am J Surg Pathol 36: 27-34, 2012.
17. Sethi R, Kozin E, Remenschneider A, Meier J, VanderLaan P, Faquin W, Deschler D and Frankenthaler R: Mammary analogue secretory carcinoma: Update on a new diagnosis of salivary gland malignancy. Laryngoscope 124: 188-195, 2014.

18. Tokuzen N, Goda H and Nakashiro K: Locally advanced mammary analogue secretory carcinoma of the parotid gland. Int J Oral Maxillofac Surg 48: 865-868, 2019.

19. Slootweg PJ, Hordijk GJ, Schade Y, van Es RJ and Koole R: Treatment failure and margin status in head and neck cancer. A critical view on the potential value of molecular pathology. Oral Oncol 38: 500-503, 2002.

20. Weiss MH, Harrison LB and Isaacs RS: Use of decision analysis in planning a management strategy for the stage N0 neck. Arch Otolaryngol Head Neck Surg 120: 699-702, 1994.

21. Shah JP, Candela FC and Poddar AK: The patterns of cervical lymph node metastases from squamous carcinoma of the oral cavity. Cancer 66: 109-113, 1990.

22. Bier J: Radical neck dissection versus conservative neck dissection for squamous cell carcinoma of the oral cavity. Recent Results Cancer Res 134: 57-62, 1994.

23. Wolff KD, Al-Nawas B, Al-Sharif U, Beck J, Bikowski K, Bissinger $\mathrm{O}$, Böhme $\mathrm{P}$, Bönte-Hieronymus $\mathrm{I}$, Bootz F, Bozzato A, et al: S3-Guidline regarding diagnosis and therapy of oral cancer. Oncology Guideline Program. AWMF, 2012 (In German). https://www.awmf.org/uploads/ tx_szleitlinien/007-100OLl_S3-Diagnostik-TherapieMundhoehlenkarzinom_2021-03.pdf. Accessed March 2021.

24. Fukuhara R, Shinya T, Fukuma S, Ogawa N, Masaoka Y, Tanaka T, Marunaka H, Arioka T, Hiraki T, Kaji M and Kanazawa S: The diagnostic capacity of pre-treatment ${ }^{18} \mathrm{~F}-\mathrm{FDG}$ PET/CT for predicting the extranodular spread of lymph node metastases in patients with oral squamous cell carcinoma. Acta Med Okayama 74: 123-128, 2020.

25. Loo SW, Geropantas K, Beadsmoore C, Montgomery PQ, Martin WM and Roques TW: Neck dissection can be avoided after sequential chemoradiotherapy and negative post-treatment positron emission tomography-computed tomography in N2 head and neck squamous cell carcinoma. Clin Oncol (R Coll Radiol) 23: 512-517, 2011.

26. Ferrell JK, Mace JC and Clayburgh D: Contemporary treatment patterns and outcomes of salivary gland carcinoma: A national cancer database review. Eur Arch Otorhinolaryngol 276: 1135-1146, 2019.

27. Amatu A, Sartore-Bianchi A and Siena S: NTRK gene fusions as novel targets of cancer therapy across multiple tumour types. ESMO Open 1: e000023, 2016.

28. Drilon A, Siena S, Ou SI, Patel M, Ahn MJ, Lee J, Bauer TM, Farago AF, Wheler JJ, Liu SV, et al: Safety and antitumor activity of the multitargeted Pan-TRK, ROS1, and ALK inhibitor entrectinib: Combined results from two Phase I Trials (ALKA-372-001 and STARTRK-1). Cancer Discov 7: 400-409, 2017.

29. Jiang T, Wang G, Liu Y, Feng L, Wang M, Liu J, Chen Y and Ouyang L: Development of small-molecule tropomyosin receptor kinase (TRK) inhibitors for NTRK fusion cancers. Acta Pharm Sin B 11: 355-372, 2021

30. Drilon A, Laetsch TW, Kummar S, DuBois SG, Lassen UN, Demetri GD, Nathenson M, Doebele RC, Farago AF, Pappo AS, et al: Efficacy of larotrectinib in TRK fusion-positive cancers in adults and children. N Engl J Med 378: 731-739, 2018.

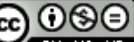

This work is licensed under a Creative Commons Attribution-NonCommercial-NoDerivatives 4.0 International (CC BY-NC-ND 4.0) License. 